\title{
EFECTO DE LA DESAMINO-OXITOCINA BUCAL SOBRE LA CONTRACTILIDAD UTERINA DURANTE LA INDUCCION DEL PARTO*
}

\author{
Aristarco Rodríguez $* *$ \\ Mery de Villamizar *** \\ Edgard Cobo ****
}

\section{La Desamino-Oxitocina (DAO)} es un derıvado sintetico de la UXItocina y presenta una potencia oxitócica superior a ésta. Su tórmula empirica es (C43 H65 O12 N11 S2), con un peso molecular de 992,2. Fue sintetizada por Lu Vigneaud y colaboradores (1). En la mujer, produce una actividad uterina dos veces mayor que la inducida por la oxitocina (10). Su acción antidiurética es mayor que la de la Oxitocina. Se inactiva con el jugo gástrico y no es destruida por la Oxitocinasa sérica (2). La actividad de la DAO se mide en unidades humanas (UH) e internacionales (UI). Las primeras se definen como la cantidad de droga necesaria para producir, administrada por vía endovenosa, la misma dinámica uterina que produce una UI de Oxitocina. La UH corresponde a $0.7 \mathrm{UI}$ del III Standard Internacional. $1 \mathrm{mgr}$. de DAO equivale a 450 UI y $450 \mathrm{UH}$. De esto se deduce que la DAO es relativamente más activa en el humano que en el animal. Las vías de administración utilizadas pueden ser la endoveno- sa, la bucal y la gástrica (3). Las dosis aconsejadas por vía bucal son mayores de $25 \mathrm{UI}$ y menores de $100 \mathrm{UI}$, a intervalos mayores de media hora.

El tiempo de latencia de la vía bucal, o sea el lapso entre su administración y la aparición de las primeras contracciones, depende de la absorción de la droga y no del tiempo de disolución, que es de treinta a cuarenta y cinco minutos. La tolerancia de la DAO por vía bucal es muy buena y los riesgos de hipertonía o sufrimiento fetal a las dosis indicadas son comparables a las de la Oxitocina endoveriosa $(4,5)$.

\footnotetext{
* Trabajo realizado en la Sección de Fisiología de la Reproducción. Departamento de Obstetricia y Ginecología. División de Salud. Universidad del Valle. Cali. Colombia.

** Profesor Auxiliar.

*** Tecnóloga de Laboratorio.

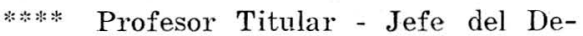
partamento.
} 


\section{Maxierial y Métodos:}

El estudio se realizó en 46 pacientes, 22 de las cuales presentaban indicaciones y condiciones para inducción del parto. Las causas de inducción fueron: Pre-eclampsia severa, isoinmunización por $\mathrm{Rh}$, rotura prematura de membranas, polihidramnios, feto muerto, embarazo prolongado y algunos casos electivos. El material se dividió en dos grupos:

Un grupo control constituido por 24 pacientes que presentaron trabajo de parto espontáneo y un grupo experimental de 22 pacientes a quienes se les practicó inducción con:DAO $50 \mathrm{UI}$ bucal con intervalos de una hora, previo enjuague de la boca con agua antes del suministro de cada tableta. En todas las pacientes el control de trabajo de parto se hizo usando el método de registro de la presión amniótica descrito por Alvarez y Caldeyro $(6,7)$. La actividad uterina se analizó midiendo el área debajo de las contracciones y se expresó en milímetros cuadrados por minuto $\left(\mathrm{mm}^{2} / \mathrm{min}\right)$ (3). Los demás parámetros de la contractibilidad del útero: intensidad, frecuencia y tono, se midieron convencionalmente (9). El progreso de la dilatación cervical y la evolución del trabajo de parto se midió por tacto vaginal; el estado fetal se observó mediante el control de la F.C.F. con Dop-tone. El protocolo de un experimento se muestra en la Fig. 1.

La calificación del estado de los recién nacidos, se midió mediante el índice de Apgar. La duración del primer período del parto, o de dilatación, se expresó en horas y en

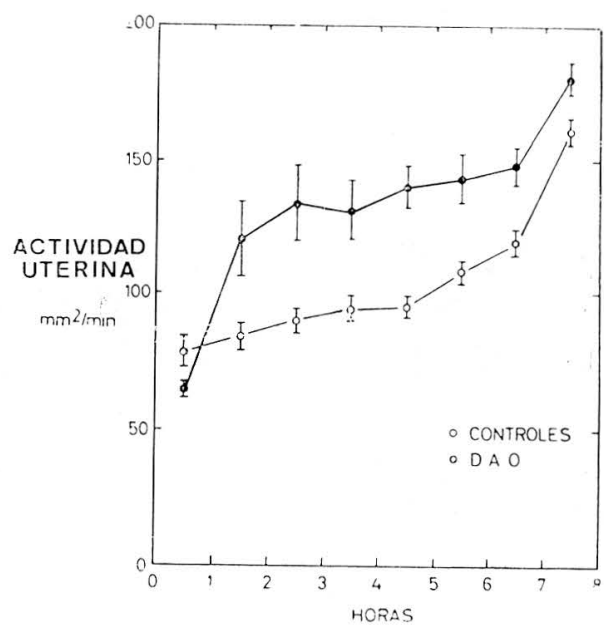

Fig. 1 Valores promedio de actividad uterina durante el parto, en casos controles y experimentales (DAO).

Obsérvese como la actividad uterina es mayor en las pacientes inducidas con DAO que en las del grupo control.

fracción de hora y la del segundo período, o expulsivo, se expresó en minutos.

Los datos encontrados se añalizaron estadísticamente haciendo promedios de los valores encontra. dos experimentalmente y buscando la significancia de las diferencias, cuando las hubo, aplicando el test de $\mathrm{t}$.

\section{Resultados:}

Las 22 pacientes del grupo experimental a quienes se les practicó inducción con DAO tuvieron 25.7 años de edad en promedio y la edad promedio de embarazo. calculada por amenorrea, fue de 39.4 semanas.

Las variedades de posición correspondieron a 15 anteriores y 7 
posteriores. En este grupo dos recién nacidos tuvieron peso promedio de $2.950 \mathrm{gr}$. y el índice de Apgar fue de 8 a 10 en 16 casos $(84.2 \%)$ y de 6 a 7 en 3 casos $(15.8 \%)$ y hubo tres casos de inducción por feto muerto retenido. (Cuadro N․ 1).

No hubo ninguna muerte fetal durante el trabajo de parto, ni intervenciones debidas a efectos producidos por la droga.

El promedio de la dosis total requerida para el parto fue de 5.7 tabletas por paciente. Las primigrávidas necesitaron en promedio 6.4 tabletas y las grávidas de 2 y más partos requirieron en promedio 5.2 tabletas.

La edad promedio de las 24 pacientes del grupo control fue 21.7 años y la edad gestacional calculada por amenorrea fue de 38.8 semanas. Las variaciones de posición correspondieron a 15 anteriores y 9 posteriores.

Los recién nacidos tuvieron un peso promedio de $3.148 \mathrm{gr}$. 22 de ellos presentaron una calificación del Apgar de 8 a $10(91.7 \%)$ y 2 una calificación de 7 . No hubo muertes fetales en este grupo:

\section{CUADDRO Nㅜ 1}

\section{Promedios de edad de embarazo, peso de los niños y Apgar en los dos grupos}

GRUPO CONTROL

38.8 semanas

Amenorrea

$3.148 \mathrm{gr}$.

22 casos $(91.7 \%)$

2.948

39.45 semanas

Peso al nacer

Apgar 8/10

$2 \operatorname{casos}(8.3 \%)$

Apgar 8/10

0

Feto Muerto

GRUPO EXPERIMENTAL

3 casos de inducción por muerte fetal.

\section{Actividad Uterina:}

Los resultados de la actividad uterina, expresada en $\mathrm{mm}^{2} / \mathrm{min}$. muestran que la contractilidad inducida por DAO fue mayor que lá cbservada en el grupo control (Figura 1).
En efecto, en el grupo control el parto se inició con una actividad de $78.5 \pm 6.9 \mathrm{~mm}^{2} / \mathrm{min}$, que fue aumentando paulatinamente hasta $119 \pm 5.2 \mathrm{~mm}^{2} / \mathrm{min}$ al final del primer período del parto, para adquirir sus máximos valores de 156士 $5.3 \mathrm{~mm}^{2} / \mathrm{min}$, durante el período expulsivo. 
En el grupo experimental en cambio, la actividad espontánea fue de $65.0 \pm 3.4 \mathrm{~mm}^{2} / \mathrm{min}$ y aumentó hasta $120.0 \pm 14.4 \mathrm{~mm}^{2} / \mathrm{min}$, con la primera dosis de DAO, subiendo progresivamente hasta IIegar a valores de $148.0 \pm 7.3 \mathrm{~mm}^{2} /$ min, al final del primer período de expulsivo llegó a valores de 180.0 $\pm 66 \mathrm{~mm}^{2} / \mathrm{min}$. La diferencia entre ambas curvas de actividad uterina fue estadísticamente significativa a partir de la tercera dosis $(\mathrm{P}<$ $0.01)$.

El efecto de la DAO sobre la actividad uterina se observó desde la primera dosis, encontrándose un

DOSIS DE D.A.O. (50 U.I.)

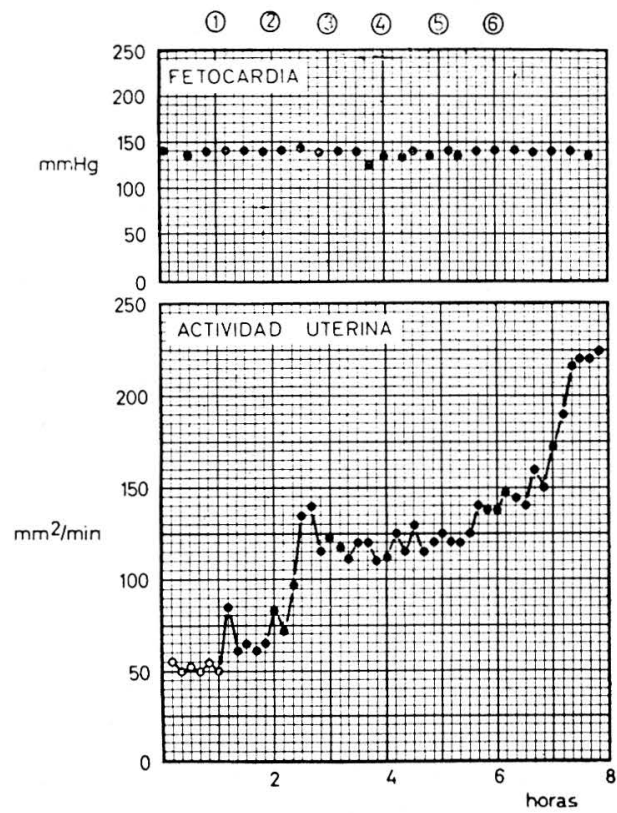

Fig. 2 Protocolo de un caso en el cual se indujo al parto con DAO. Las dosis se numeran por orden de administración dentro de los círculos superiores. Obsérvese como la fetocardia y tensión arterial no presentan mayores período de latencia de $19.8 \pm 2.26$ minutos en 20 casos; solo dos respondieron después de la segunda dosis. A partir del efecto observado, la actividad uterina continuó aumentando progresivamente hasta la expulsión del feto. Sin embargo, se observó que el mayor aumento de la actividad uterina ( $\triangle \mathrm{AU}$ ) ocurrió con la primera dosis (A $A . U=55 \mathrm{~mm}^{2} / \mathrm{min}$.) y que los aumentos sucesivos con cada una de las dosis fueron menores, del orden de $5 \mathrm{~mm}^{2} / \mathrm{min}$; esto quiere decir, que una veź obtenido el efecto inicial no ocurren aumentos mayores en el curso del trabajo de parto (Fig. 2).

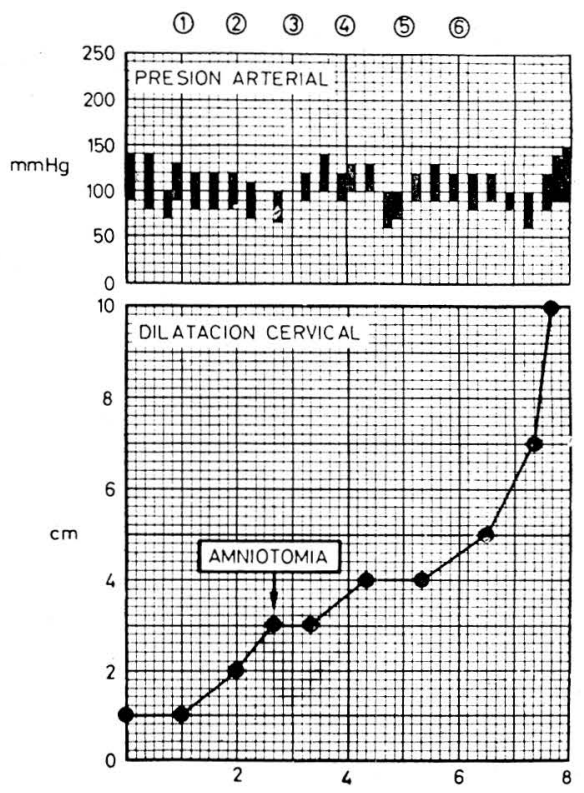

variaciones durante el trabajo de parto. La actividad uterina aumenta casi bruscamente a partir de la $2^{\text {a hora } y}$ luego continúa haciéndolo muy discretamente. La dilatación uterina sigue una evolución normal. 


\section{Iriterisidad:}

La intensidad (expresada en $\mathrm{mm}$ $\mathrm{Hg}$ ) inducida por la DAO fue mavor que la observada en el grupo control. En este grupo, el trabajo de parto tuvo una intensidad inicial de $28 \pm 3.1 \mathrm{~mm} \mathrm{Hg}$ de dilatación para ascender a $38 \pm 1.6$ minutos al final del período y a $42 \pm 2.06$ $\mathrm{mm} \mathrm{Hg}$ en el período expulsivo. En el grupo experimental en cambio, la intensidad espontánea fue de $27 \pm 3.5 \mathrm{~mm} \mathrm{Hg}$ y aumentó hasta $37.1 \pm 3.1 \mathrm{~mm} \mathrm{Hg}$ con la primera dosis, subiendo progresivamente hasta llegar a valores de 49.6士 $3.1 \mathrm{~mm} \mathrm{Hg}$ al final del primer período del parto y alcanzar sus máximos valores de $53.4 \pm 3.1 \mathrm{~mm}$ $\mathrm{Hg}$ durante el período expulsivo. Los valores promedios de la intensidad en ambos grupos, no son muy diferentes hasta la cuarta ho. ra, a partir de la cual el aumento de la intensidad en el grupo experimental es mayor y estadísticamente significativo, continuando así hasta el final del parto. El aumento sianificativo de la actividad uteriria, descrita atrás, está claramente relacionado con el aumento de la intensidad de las contracciones uterinas que acabamos de describir (Fig. 3). En la figura 4, se compara la contractilidad uterina registrada en uno de los casos del grupo control, con la registrada en uno de los casos inducidos con DAO; se observa claramente como la intensidad de las contracciones es muy alta, presentando frecuentemente valores de $90 \mathrm{~mm} \mathrm{Hg}, 0$ más.

\section{Frecuencia y Tono:}

Tarito la frecuencia, como el tono, siguieron una evolución simi-

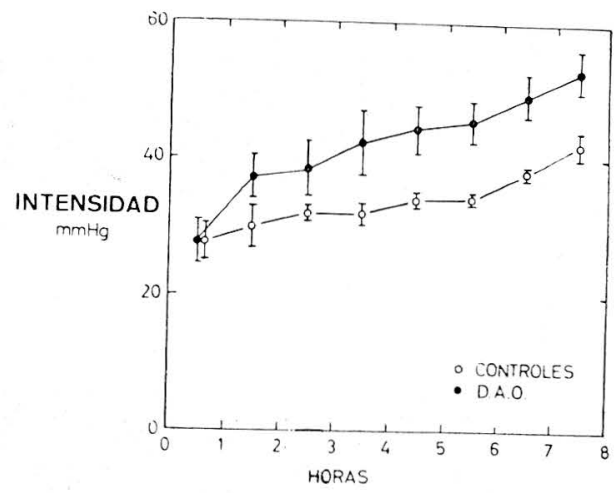

Fig. 3 Valores promedio de la intensidad de las contracciones, en casos controles y experimentales (DAO).

lar en los casos inducidos con $\mathrm{DAO}$, como los del grupo control.

La frecuencia (expresada en número de contracciones producidas en 10 minutos) del grupo control fue de $3.6 \pm 0.7 \mathrm{C} / 10 \mathrm{~min}$.

En el grupo experimental la frecuencia fue de $3.7 \pm 1.2 \mathrm{C} / 10 \mathrm{~min}$. durante la primera hora, de $5.0 \pm$ $0.2 \mathrm{C} / 10 \mathrm{~min}$. al final del primer período y de $5.2 \pm 0.2 \mathrm{C} / 10 \mathrm{~min}$. durante el período expulsivo (Fig. 5) .

Los valores promedio del tono (expresados en $\mathrm{mm} \mathrm{Hg}$ ) fueron en el grupo control de $10.0 \pm 1.1 \mathrm{~mm}$ $\mathrm{Hg}$ durante la primera hora, de $11.0 \pm 0.6 \mathrm{~mm} \mathrm{Hg}$ al final del primer período, y de $13.0 \pm 0.8 \mathrm{~mm}$ $\mathrm{Hg}$ durante el período expulsivo. Fueron similares a los del grupo inducido ccn DAO que mostró 8.0 $\pm 1.7 \mathrm{~mm} \mathrm{Hg}$ para la primera ho. ra, 9.4 $\pm 0.5 \mathrm{~mm} \mathrm{Hg}$ para el final del período de dilatación y $11.1 \pm$ $0.4 \mathrm{~mm} \mathrm{Hg}$ en el período expulsivo (Fig. 5). 


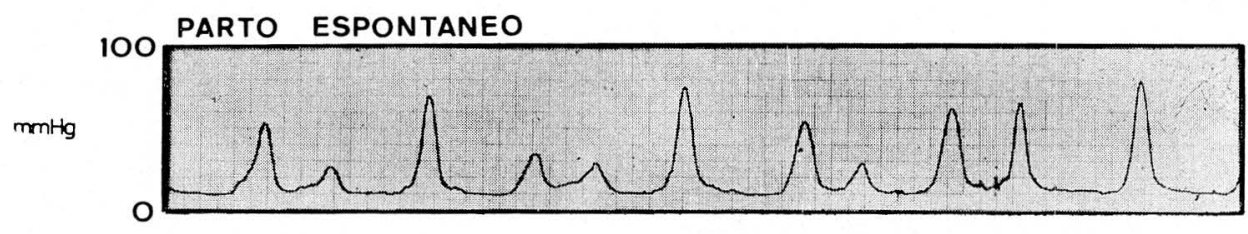

\section{PARTO INDUCIDO CON DESAMINO-OXITOCINA}

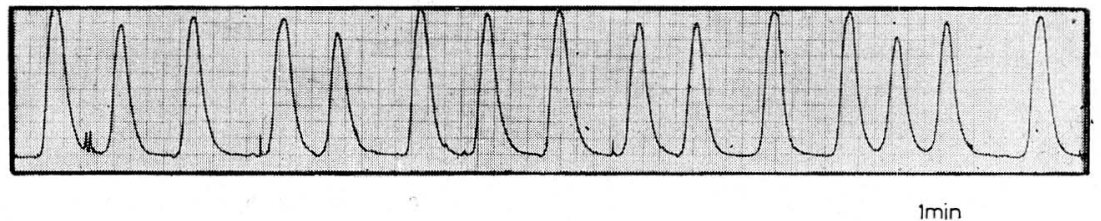

Fig. 4 Comparación de dos registros de presión amniótica correspondientes a un parto espontáneo y a uno inducido con DAO.

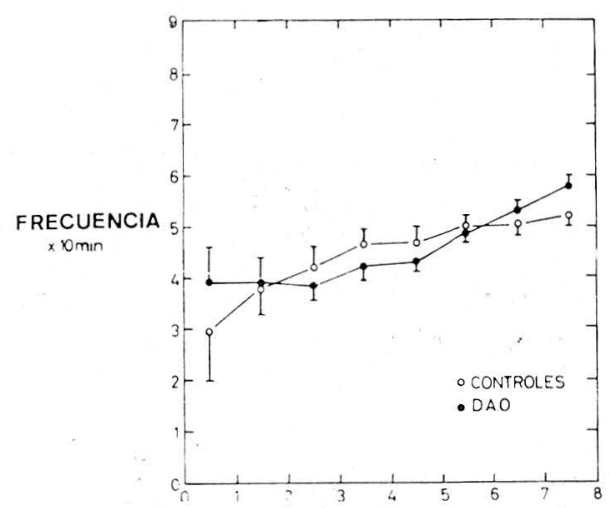

Fig. 5 Valores promedio de frecuencia (izquierda) y tono (derecha), durante el parto, en casos controles y experimentales (DAO).

Las pequeñas diferencias encontradas no fueron estadísticamente significativas. En dos casos se presentaron polisistolias entre $5.0 \mathrm{y}$ 7.7 contracciones por 10 minutos,
Obsérvese como el aumento de la actividad en el parto inducido se hizo a expensas de la intensidad, más que de la frecuencia de las contracciones.

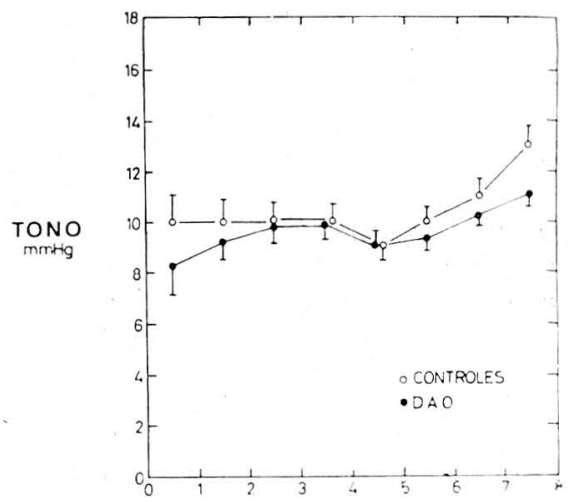

Obsérvese como las curvas de frecuencia y tono en ambos grupos de estudio no presentan mayores diferencias.

los cuales fueron de muy corta duración y cedieron sin necesidad de interrumpir la administración de la droga. 


\section{Efecto de la Rotura de Membranas}

En los 17 casos en los cuales se practicó amniotomía, se encontró un aumento de la actividad uterina durante la primera hora que siguió a este procedimiento; dicho aumento fue producido por elevaciones tanto de la frecuencia como de la intensidad de las contracciones.

\section{Duración del Parto}

La duración del parto la expresamos en horas y fracción de hora para el período de dilatación y en minutos para el período expulsivo. Además, las pacientes fueron clasificadas en primigrávidas y en pacientes con 2 y más partos.

\section{DILATACION}
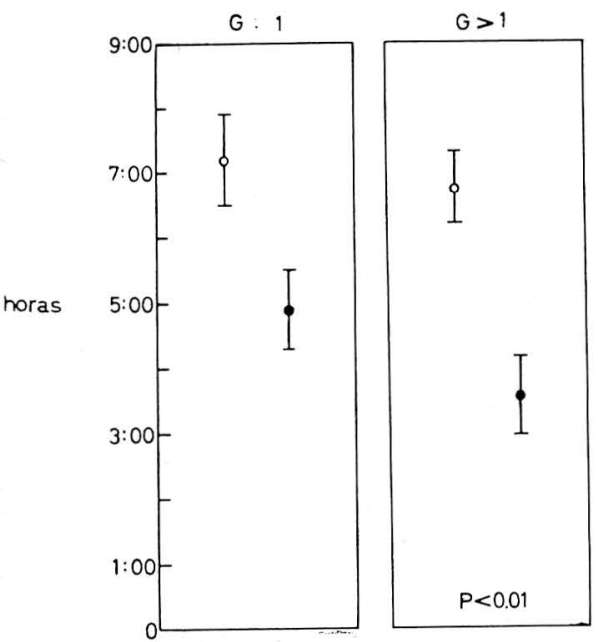

Fig. 6 Duración de los períodos de dilatación y expulsivo, en ambos grupos de estudio. Se compara la duración en horas del periodo de dilatación en pacientes controles y del grupo experimental, subdivididas en primigrávidas y con
La duración del parto fue sensiblemente mayor para el grupo control que para el experimental (Fig. $6)$. Las primíparas del grupo control (Fig. 6, izquierda) emplearon para el período de dilatación 7.2 horas y las del grupo experimental 4.8 horas, $(P<0.02)$. Las de 2 y más partos emplearon 6.7 horas y 3.5 horas para los grupos control y experimental respectivamente; estas diferencias son estadística. mente significativas $(P<0.01)$.

Durante el período expulsivo, los valores para primigrávidas del grupo control fueron de $33.7 \pm 5.2 \mathrm{mi}^{-}$ nutos y de $18.4 \pm 3.3$ minutos para el grupo experimental; esta diferencia fue estadísticamente significativa $(P<0.02)$, en cambio, las

\section{EXPULSIVO}
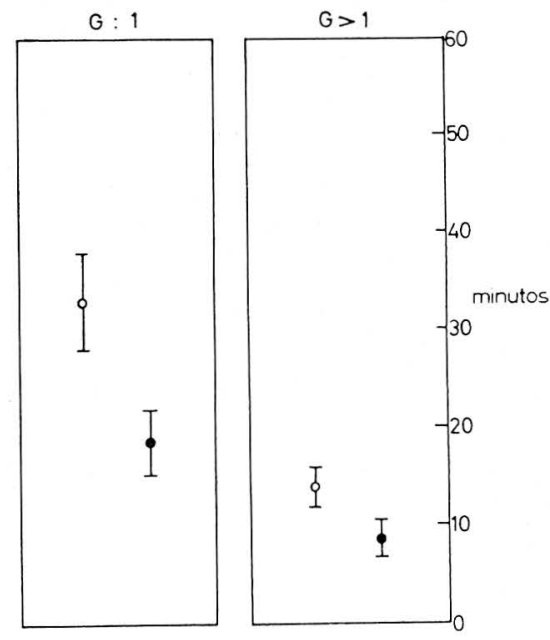

- CONTROLES
- DA.O.

2 y más partos. Así mismo, se compara la duración en minutos del período expulsivo. Obsérvese como la duración de estos períodos es más corta en las pacientes inducidas con DAO. 
de 2 y más partos gastaron 14.1士 2 minutos y $8.7 \pm 1.9$ minutos para los grupos control y el inducido con DAO respectivamente, no siendo la diferencia estadísticamente significativa (Fig. 6, derecha).

\section{Discusión}

Las pacientes sometidas a inducción con DAO tuvieron en su mayoría indicaciones precisas entre las que figuraron 6 toxémicas y 5 por rotura prematura de membranas, causas o factores que pudieron influir favorablemente en el desarrollo del trabajo de parto, no así las del grupo control que no presentaron patología alguna.

Nuestros resultados demuestran que la actividad uterina inducida con DAO, es significativamente mayor que la del parto esporitáneo a expensas del aumento de la intensidad de las contracciones. Estos resultados son comparables a los obtenidos por Massi y colaboradores (10), quienes estudiaron el efecto de infusiones endovenosas de DAO y de Oxitocina sintética, encontrando que con dosis de 2 a $6 \mathrm{mg} \times 10^{-6} / \mathrm{min} / 60 \mathrm{~kg}$, el efecto de la DAO sobre la actividad uterina es 2.66 veces mayor que el de la Oxitocina sintética.

Esta diferencia fue producida primordialmente por un aumento de la intensidad de las contracciones.

Sin embargo, debe destacarse que, a pesar del sensible aumento de la actividad uterina, el tono uterino se mantiene dentro de límites normales.
La duración del período de dilatación fue significativamente menor en las pacientes que recibieron DAO, bien fueran ellas primigrávidas o con 2 ó más partos. De la misma manera, la duración del período expulsivo fue significativamente menor en las primigrávidas inducidas con DAO. Estos hallazgos pueden relacionarse con la actividad uterina mayor, producida por esta substancia.

Los resultados obtenidos en la calificación del Indice de Apgar muestran que en los Recién Naci dos del grupo experimental, este es ligeramente inferior al del grupo control, pero debe tenerse en cuenta que en dicho grupo experimental se estudiaron algunos casos con patología asociada, como Toxemia del Embarazo, lo cual no ocurrió en el grupo control. Esta diferencia, podría explicar la disminución del Indice de Apgar en el grupo que recibió DAO.

Por esta razón creemos que no debe atribuirse al exceso de actividad uterina inducida por la DAO el hallazgo de algunos Indices de A.pgar ligeramente bajos en este grupo, más aún, si se tiene en cuenta que el grupo control no presentó ningún tipo de patología.

Como es aconsejable tratar de reproducir, en lo posible, la contractilidad propia del parto normal, y no una actividad mayor como ocurre con la DAO, sugerimos que en aquellos casos de actividad muy alta, se disminuya la dosis a media tableta cada hora. Finalmente, debemos destacar que la DAO en nuestra experiencia constituyó un método seguro y cómodo de inducción del parto. 


\section{Resumen:}

Se estudian los efectos sobre el útero humano de la oxitocina sintética (DAO); la actividad uterina se mide por registros de presión intra-amniótica. El estudio com. prende 24 embarazadas normales a término con trabajo espontáneo como grupo de control, y 22 en quienes la inducción electiva tiene causas médicas, como grupo de control. Tabletas con 50 unidades de la droga aplicaron cada hora, en el surco gíngivo-labial.

Se verificó aumento significativo de la actividad uterina después de la tercera tableta y el aumento se debe principalmente a la mayor intensidad de la contracción.

La duración del primer período del parto fue más corta en el grupo experimental. No hubo diferencias en los recién nacidos de uno u otro grupo. Sin embargo, aconsejamos precaución en el manejo de la (DAO).

\section{Summary:}

The effects of a syntetic analogue of oxytocin, namely deaminooxytocin (D.A.O.) upon the pregnant human uterus, were studied. Intra-amniotic pressure was recorded as a method of measuring uterine activity. The fetal and neonatal status was clinically monitored. The study comprises 24 normal term pregnant women in whom spontaneous labor was recorded (control group) and 22 to whom interruption of pregnancy was performed electively or for medic al reasons (experimental group). 50 I. Units of D.A.O., con- tained in a tablet were administered every hour via the gingivo-labial route.

Results showed a significant increase of uterine activity after the administration of the third D.A.O. 's tablet. This increase in uterine action ocurred mainly because of the high contraction intensity induced by D.A.O.

Lenght of the first stage of labor was shorter in the experimental than in the control group. No sigrificant differences were observed between the newborns corresponding to the control and the experimental group, in spite of increased uterine activity and short labor in the later group. Nevertheless, it is our feeling that some caution must be exercised when mothers of weights and heights lower than average are selected for D.A.O.'s induction. In those cases, a dose of 25 I. Units (half tablet) every hour may be enough to induce labor.

\section{REFERENCIAS}

1. Du Vigneaud, V., Winestock, C. Murti, V. V. S., Hope, D. B. y Kimbrough, R. D.: "Synthesis of 1-B mercapto propionic acid Oxytocin (desamino-oxitocin) a Highly Potent Analogue of Oxytocin". J. Biol. Chem.

2. Hope, D. B. Murti, V. V. S. and Du Vigneaud, V.: "A Highly Potent Analogue of Oxytocin, DesaminoOxytocin". J. Biol. Chem. 237:1563, 1962

3. Embrey, M. P.: "The Action of Desamino-Oxytocin on the Human 
Pregnant Uterus". J. Endocr. 31: 185, 1965.

4. Berger, M. : "Administration Transbuccale d'Ocytocine et d'un Derive de la Ocytocine". Praxis 57:588, 1968.

5. Mendes, M. L,: "Desamino-oxitocina em Clinica Obstétrica". Coimbra Míd) 16:439, 1969.

6. Alvarez, H. y Caldeyro-Barcia, R.: "Nueva técnica para registrar la Actividad Contractil del Utero $\mathrm{Hu}$ mano". Arch. Ginec. y Obst. 9:1, 1951.

7. Caldeyro-Barcia, R. and Poseiro, J. J.: "Physiology of the Uterine Contraction". Clin. Obst. \& Gynec. $3: 386,1960$.
8. Cobo, E.: "Uterine and Mil-ejecting Activities during Human Labor". J. Appl. Physiol. 24:317, 1968.

9. Caldeyro-Barcia, R., Sica-Blanco, y., Poseiro, J. J., Gonzales-Panizza, V., Mendez-Baver, C., Fielitz, C., Alvarez, H., Pose, S. V., and Hendricks C. H.: "A Quantitative Study of the Action of Synthetic Oxytocin on the Pregnant Human Uterus". J. Pharmacol \& Exper. Therap. 121:18, 1957.

10. C. B. Massi, J. J. Poseiro R. Caldeyro-Barcia, V. Du Vigneaud.: "Effetti della Desaminoossitocina sulla Contrattilita dell Utero Umano a Termine di Gravidanza". Riv. Ostet. Ginec. 20,77-86, 1965. 